\title{
Compositeness above the electroweak scale and a proposed test at $\mathrm{LHCb}$
}

\author{
Gabriele Ferretti ${ }^{1, *}$ \\ ${ }^{1}$ Department of Physics, Chalmers University of Technology, Fysikgården, 41296 Göteborg, Sweden
}

\begin{abstract}
I review attempts to construct models of partial compositeness from strongly coupled gauge theories. A few minimal assumptions allow one to isolate a small number of representative models. After presenting the main idea, I discuss a recent proposal to detect a light pseudo-scalar, predicted in all these models, at the $\mathrm{LHCb}$ detector.
\end{abstract}

\section{Introduction}

The Higgs boson is nine years old but shows none of the restless behavior typical of that age, obeying all the rules of the Standard Model (SM). Still, many of us hope that it will eventually help us breaking the shackles of the SM and lead us into a new world. The reason for this hope is, of course, that its mass is not "Natural" and I still unapologetically embrace this argument.

There are basically two symmetry-based ways of attacking this problem. The first one is supersymmetry, pairing the Higgs field $h$ with a fermionic field $\psi$ whose mass is protected by chiral symmetry. In this case the pairing occurs via the fermionic transformation $\delta h \approx \epsilon \psi$ and the Higgs mass is protected indirectly via the fermion mass. The second mechanism employs a regular bosonic symmetry, may that be a scale $\delta h \approx \lambda h$ or a shift symmetry $\delta h \approx h+a$ protecting directly the Higgs mass. The last case arises in models where the Higgs is a composite (pseudo-)Nambu-Goldstone boson (pNGB) of a spontaneously broken approximate global symmetry [1].

Supersymmetry is a weakly coupled theory with natural elementary scalars and its problem is that it predicts too much in the sense that there are many unwanted $\operatorname{dim}<4$ operators that need to be suppressed somehow. On the contrary, compositeness is a strongly coupled theory without elementary scalars that predicts too little, in the sense that we lack the dim = 4 Yukawa operators needed to give fermion masses.

In this talk I concentrate on some model of compositeness, with the Higgs boson arising as a pNGB. While one should remain aware of the above central difficulty for all these constructions, one can at least argue that Nature already does make use of this mechanism in the description of pion and kaon dynamics with chiral lagrangians. It seems a shame not to use this idea more often!

*e-mail: ferretti@chalmers.se 


\begin{tabular}{|c|c|}
\hline $4(\psi, \tilde{\psi}) \in$ Complex irrep & $S U(4) \times S U(4)^{\prime} / S U(4)_{D}$ \\
\hline $4 \psi \in$ Pseudoreal irrep & $S U(4) / S p(4)$ \\
\hline $5 \psi \in$ Real irrep & $S U(5) / S O(5)$ \\
\hline
\end{tabular}

Table 1. Minimal fermionic content for realistic EW symmetry breaking. All fermions are thought of as left-handed Weyl spinors.

\section{Basic idea}

The basic idea is to start with the Higgsless (and thus massless) part of the SM lagrangian

$$
\mathcal{L}_{\mathrm{SM} 0}=-\frac{1}{4} \sum_{F=G W B} F_{\mu \nu}^{2}+i \sum_{\Psi=Q u d L e} \bar{\Psi} \not D \Psi
$$

and couple it to a theory $\mathcal{L}_{\text {comp. }}$ with hyper-color gauge group $G_{\mathrm{HC}}$ and global symmetry structure $G_{\mathrm{F}}$ at some "flavor" scale $\Lambda_{\mathrm{UV}} \geq 10^{3} \mathrm{TeV}$ spontaneously broken to $G_{\mathrm{F}} \rightarrow H_{\mathrm{F}}$ at energies $\Lambda$ of a few $\mathrm{TeV}$ such that the Higgs doublet $H \in G_{\mathrm{F}} / H_{\mathrm{F}}$ and

$$
\mathcal{L}_{\text {comp. }}+\mathcal{L}_{\mathrm{SM} 0}+\mathcal{L}_{\text {int. }} \longrightarrow \mathcal{L}_{\mathrm{SM}}+\cdots
$$

(where $\mathcal{L}_{\mathrm{SM}}+\cdots$ is the full SM plus possibly extra light matter from bound states of $\mathcal{L}_{\text {comp. }}$ at energies below $\Lambda$.)

In order to realize the symmetry breaking mechanism above without reincurring in problems with naturalness one is led to use a fermionic bilinear condensate. The main difference with technicolor models is that the vacuum condensate is only mildly misaligned w.r.t. the electroweak (EW) group leading to acceptable corrections to the S-parameter. As far the EW sector is concerned, the minimal cosets of this type are those in table 1. The required misalignment can be induced by coupling the SM fermions to the hyper-color theory [2]. In order to accomplish this, one introduces in $\mathcal{L}_{\text {int. }}$ four-fermion couplings between one SM fermion and three hyper-fermions, effectively giving rise, at low energies, to a linear coupling between SM fermions and composite bound-states from the strongly-coupled sector carrying the same SM quantum numbers as the SM fermion. This also gives rise to a novel mechanism to generate fermion masses known as partial compositeness [3]. A convenient way to realize this $[4,5]$ is to introduce two different types of hyper-fermions $\psi$ and $\chi$ transforming under different irreps of $G_{\mathrm{HC}}$. The $\psi \mathrm{s}$ are the same fermions that were employed to construct the EW coset of table 1, while the $\chi$ s carry the necessary QCD color quantum numbers to allow coupling to the quarks, most notably the top quark.

In previous work $[6,7]$, we narrowed down the possibilities to a set of twelve models, summarized in table 2 . The models in table 2 were selected (using the multi-loop betafunction) as those likely to lay outside of the conformal window, and thus in the confining region. The rationale for this choice is that one can always add more degrees of freedom to bring the theory inside the conformal window at a higher scale. If the scaling dimension of the fermionic composite operator is sufficiently large this allows the SM fermion mass (most crucially that of the top quark) to remain sizable until the confinement scale $\Lambda$ is reached. Below this scale, these additional degrees of freedom decouple and the theory reduces to that described in table 2 giving rise to composite resonances at the $\mathrm{TeV}$ scale, among which the top partners. 


\begin{tabular}{|c|c|c|c|}
\hline$G_{\mathrm{HC}}$ & $\psi$ & $\chi$ & $G_{\mathrm{F}} / H_{\mathrm{F}}$ \\
\hline$S O(7)$ & $5 \times \mathbf{F}$ & $6 \times$ Spin & \multirow{4}{*}{$\frac{S U(5)}{S O(5)} \frac{S U(6)}{S O(6)} U(1)$} \\
\hline$S O(9)$ & $5 \times \mathbf{F}$ & $6 \times$ Spin & \\
\hline$S O(7)$ & $5 \times$ Spin & $6 \times \mathbf{F}$ & \\
\hline$S O(9)$ & $5 \times$ Spin & $6 \times \mathbf{F}$ & \\
\hline$S p(4)$ & $5 \times \mathbf{A}_{2}$ & $6 \times \mathbf{F}$ & $\frac{S U(5)}{S O(5)} \frac{S U(6)}{S p(6)} U(1)$ \\
\hline$S U(4)$ & $5 \times \mathbf{A}_{2}$ & $3 \times(\mathbf{F}, \overline{\mathbf{F}})$ & \multirow{2}{*}{$\frac{S U(5)}{S O(5)} \frac{S U(3) \times S U(3)^{\prime}}{S U(3)_{D}} U(1)$} \\
\hline$S O(10)$ & $5 \times \mathbf{F}$ & $3 \times($ Spin,$\overline{\text { Spin }})$ & \\
\hline$S p(4)$ & $4 \times \mathbf{F}$ & $6 \times \mathbf{A}_{2}$ & \multirow{2}{*}{$\frac{S U(4)}{S p(4)} \frac{S U(6)}{S O(6)} U(1)$} \\
\hline$S O(11)$ & $4 \times$ Spin & $6 \times \mathbf{F}$ & \\
\hline$S O(10)$ & $4 \times($ Spin,$\overline{\text { Spin }})$ & $6 \times \mathbf{F}$ & \multirow{2}{*}{$\frac{S U(4) \times S U(4)^{\prime}}{S U(4)_{D}} \frac{S U(6)}{S O(6)} U(1)$} \\
\hline$S U(4)$ & $4 \times(\mathbf{F}, \overline{\mathbf{F}})$ & $6 \times \mathbf{A}_{2}$ & \\
\hline$S U(5)$ & $4 \times(\mathbf{F}, \overline{\mathbf{F}})$ & $3 \times\left(\mathbf{A}_{2}, \overline{\mathbf{A}}_{2}\right)$ & $\frac{S U(4) \times S U(4)^{\prime}}{S U(4)_{D}} \frac{S U(3) \times S U(3)^{\prime}}{S U(3)_{D}} U(1)$ \\
\hline
\end{tabular}

Table 2. Fermionic content of the twelve models of strongly coupled gauge theories passing the basic requirements of giving rise to a composite Higgs sector respecting custodial symmetry and a top quark partner. The models are referred to as M1 through M12. These models are conjectured to be outside of the conformal window.

\section{Questions for the Lattice}

In trying to estimate the viability of these models the first questions to be addressed concern the composite sector in isolation, before coupling it to the SM. For this task, the lattice can provide invaluable information. Looking at table 2 from this point of view one sees that the models can be grouped into three main classes:

- $S U(4)$ with $N_{F}$ Fundamentals and $N_{A}$ Antisymmetric (possibly also $S U(5)$ )

- $S p(4)$ with $N_{F}$ Fundamentals and $N_{A}$ Antisymmetric

- $S O(N)$ with $N_{F}$ Fundamentals and $N_{S}$ Spin (with $N=7,9,10,11$ )

Lattice simulations of models in the first two classes have been performed (e.g. [8-11]) and more are underway. In figure $1 \mathrm{I}$ give a schematic overview of the positioning of the four models of table 2 involving the hyper-color groups $S p(4)$ and $S U(4)$. The positioning of the lower edge of the conformal window is an educated guess at best, but one can see that the models of interest fall very close to it, raising the hope that strong coupling effects give rise to the large anomalous dimensions needed for a realistic top quark mass.

Among the questions that one would like to answer are: Where does the boundary of the conformal window start? For models inside the window, can one find a fermionic composite operator of scaling dimension $\approx 5 / 2$ ? What are the masses of the composite fermionic resonances for the models in table 2? Can one estimate the low energy coefficients in the pNGB potential? Can one estimate the top Yukawa coupling? Some of these questions are starting to be addressed for $S U(4)[8,9]$ and $S p(4)[10,11]$. 

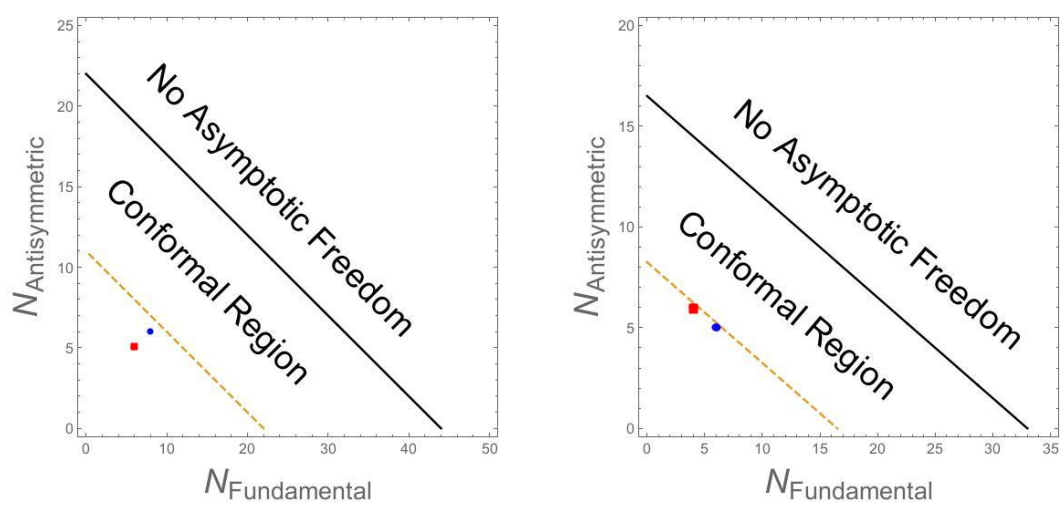

Figure 1. Positioning of some of the models in figure 2. Left: $G_{\mathrm{HC}}=S U(4)$ (red square=M6, blue dot=M11). Right: $G_{\mathrm{HC}}=S p(4)$ (red square=M8, blue dot=M5). All fermions are counted as Weyl fermions.

\section{An additional light ALP}

In this section I describe a common feature $[12,13]$ of all of these models showing some promising potential for exclusion/discovery in hadronic collisions, most notably at $\mathrm{LHCb}$ [14]. At the classical level, all models in table 2 posses two axial $U(1)$ symmetries that are spontaneously broken by the hyper-quark condensates and weakly explicitly broken by the coupling to the SM fields. We can denote these two symmetries by $U(1)_{\psi}$ and $U(1)_{\chi}$ acting uniformly on all the flavor and gauge components of the fields, e.g. $\psi \rightarrow e^{i \alpha} \psi$. In the case of $\psi$ transforming under a complex representation of $G_{\mathrm{HC}}$ this symmetry acts in the same way on the conjugate Weyl spinor as well: $(\psi, \tilde{\psi}) \rightarrow\left(e^{i \alpha} \psi, e^{i \alpha} \tilde{\psi}\right)$. In this case there is an additional vector like symmetry $(\psi, \tilde{\psi}) \rightarrow\left(e^{i \beta} \psi, e^{-i \beta} \tilde{\psi}\right)$, unbroken by the condensate $\langle\tilde{\psi} \psi\rangle$, that does not lead to additional pNGBs and shall not be discussed further.

Both axial $U(1)_{\psi, \chi} \mathrm{s}$ are subjected to a $U(1)_{\psi, \chi} G_{\mathrm{HC}} G_{\mathrm{HC}} \mathrm{ABJ}$ anomaly, but one linear combination remains anomaly free. Denoting by $q_{\psi, \chi}$ the $U(1)$ charges of this combination, $N_{\psi, \chi}$ the number of flavor components (always counting Weyl degrees of freedom) and $T_{\psi, \chi}$ the index of their $G_{\mathrm{HC}}$ irrep $\left(T_{\psi, \chi}=T_{\tilde{\psi}, \tilde{\chi}}\right.$ for complex irreps) we require $q_{\psi} N_{\psi} T_{\psi}+q_{\chi} N_{\chi} T_{\chi}=0$ for the cancellation of the anomaly. The pNGB $a$ associated to the breaking of this combination $U(1)_{a}$ is light, since its mass comes from the explicit terms coupling the hyper-color theory to the SM. Let us denote the mass and "decay constant" of this pNGB by $m_{a}$ and $f$ respectively.

After canceling the $U(1)_{a} G_{\mathrm{HC}} G_{\mathrm{HC}}$ anomaly, there remains non-zero $U(1)_{a} S U(3) S U(3)$ and $U(1)_{a} U(1)_{\text {e.m. }} U(1)_{\text {e.m. }}$ anomalies with the QCD and QED gauge group, thus warranting calling this pNGB an "axion-like" particle (ALP). However, I hasten to point out that, because of the presence of the explicit breaking terms, this particle cannot solve the strong CP problem of QCD. For the same reason, its mass $m_{a}$ is freed from obeying the relation $f m_{a} \approx f_{\pi} m_{\pi}$ typical of the proper QCD axion. This allows it to evade the usual constraints that exclude axions with decay constants $f<10^{11} \mathrm{GeV}$. Needless to say, this particle decays promptly and has no relevance to Dark Matter. For an ALP decay constant $f=1 \mathrm{TeV}$, for the models in table 2, the widths are in the $\mathrm{MeV}$ range for masses $m_{a} \approx 60 \mathrm{GeV}$, decreasing to few $\mathrm{keV}$ for masses $m_{a} \approx$ few $\mathrm{GeV}$.

Because of its coupling to the gluon, this ALP could be the target of searches at hadron colliders. While the mass and decay constant depend on the strong coupling physics and on the specific details of the explicit symmetry breaking, the remaining couplings to the SM 
fermions and gauge bosons are more under control and can be estimated form the data in table 2. This leads to the following phenomenological lagrangian whose only undetermined parameters are $m_{a}$ and $f$.

$$
\mathcal{L}_{\mathrm{eff}}=\frac{1}{2}\left(\partial_{\mu} a\right)^{2}-\frac{1}{2} m_{a}^{2} a^{2}-i \sum \frac{C_{\Psi} m_{\Psi}}{f} a \bar{\Psi} \gamma^{5} \Psi+\frac{a}{16 \pi^{2} f}\left(g_{s}^{2} K_{g} G_{\mu \nu}^{a} \tilde{G}^{a \mu \nu}+e^{2} K_{\gamma} F_{\mu \nu} \tilde{F}^{\mu v}\right) .
$$

In (3) the sum is over the SM fermions $\Psi$ of mass $m_{\Psi}, G_{\mu \nu}^{a}$ and $F_{\mu \nu}$ are the QCD and QED field strength and $g_{s}$ and $e$ their coupling constants. The couplings in (3) for the various models can be found in [14].

The strongest current bounds at low masses $m_{a}<60 \mathrm{GeV}$ come from the $a \rightarrow \mu^{+} \mu^{-}$ channel and are given in [15]. They are presented as bounds on the mixing angle $\theta_{H}$ of the type IV two Higgs doublet model (2HDM) of $[16,17]$ at $\tan \beta=0.5$. They can be easily recast to the models presented here by the following observation. In the case of the 2HDM models, both the production cross-sections and the decay rates are proportional to $\sin ^{2} \theta_{H}$, while in the present models they scale as $(v / f)^{2}$, with $v=246 \mathrm{GeV}$ the Higgs vacuum expectation value. This means that the recast can be easily obtained by the formula

$$
\frac{v}{f}=\sqrt{\frac{\sigma\left(p p \rightarrow a \rightarrow \mu^{+} \mu^{-}\right)_{\sin \theta_{H}=1}}{\sigma\left(p p \rightarrow a \rightarrow \mu^{+} \mu^{-}\right)_{v=f}}} \sin \theta_{H},
$$

where the cross-sections are those of the 2HDM and (3) in the numerator and denominator respectively. The results of this recast are shown in figure 2, taken from [14].

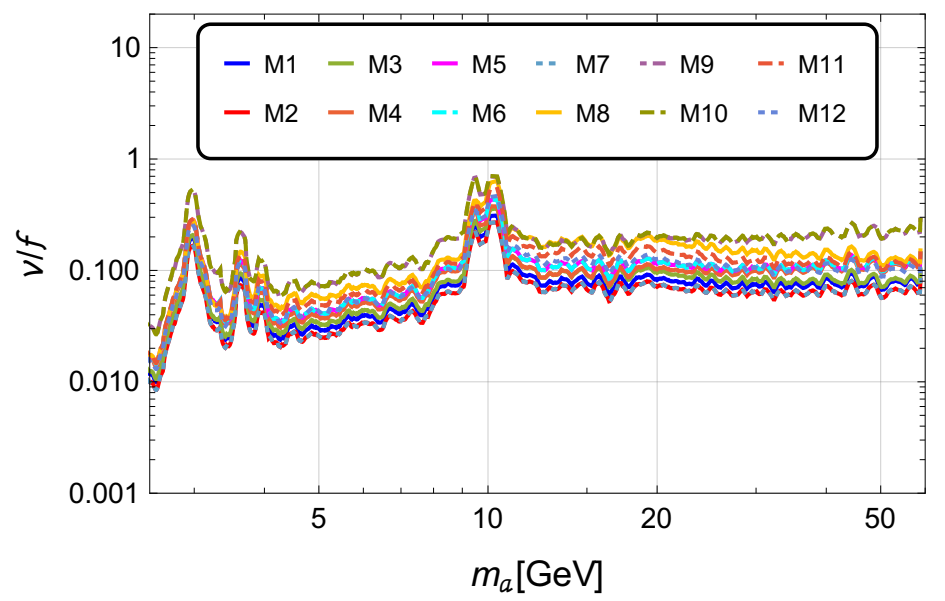

Figure 2. Recast of the LHCb bounds [15] for the present models. Taken from [14].

$\mathrm{LHCb}$ has also great potential to investigate other channels that have so far not been studied. One such channel is the $a \rightarrow \tau^{+} \tau^{-}$decay mode, which is enhanced by a factor $\left(m_{\tau} / m_{\mu}\right)^{2} \approx 286$ compared to the di-muon channel, under the assumption of $C_{\tau} \approx C_{\mu}$ in (3). The gain from this enhancement is however mitigated by the well known difficulties of working with taus, namely the unavoidable presence of missing energy (due to neutrinos) and the presence of hadronic tau decays that suffer from a large background. While all tau decay modes deserve attention, in [14] we showed that the most sensitive final state is the one in which the two taus decay to an electron and a muon respectively (the so-called opposite sign 
opposite flavor mode, (OSOF)). This comprises only $6.2 \%$ of the available final states from tau pairs but has the advantage of allowing for a significant reduction of the background at LHCb.

The full details of the analysis of the OSOF channel are given in [14]. Here I limit myself to point out that the two main sources of background for this mode are Drell-Yan production $\left(p p \rightarrow \tau^{+} \tau^{-}\right)$and bottom pairs production $(p p \rightarrow b \bar{b})$. The light (including charm) QCD jet background, which is the leading source of background for the semi-leptonic and hadronic tau decays is subdominant in this case. As an example, with the cut-flow discussed in [14], the signal efficiency for $m_{a}=20 \mathrm{GeV}$ is $1.08 \times 10^{-3}$, while the Drell-Yan production is suppressed by $1.20 \times 10^{-4}$ and $b \bar{b}$ production by $2.65 \times 10^{-9}$.

The projected sensitivity in $v / f$ at $15 \mathrm{fb}^{-1}$ is shown in figure 3. Comparing the two anal-

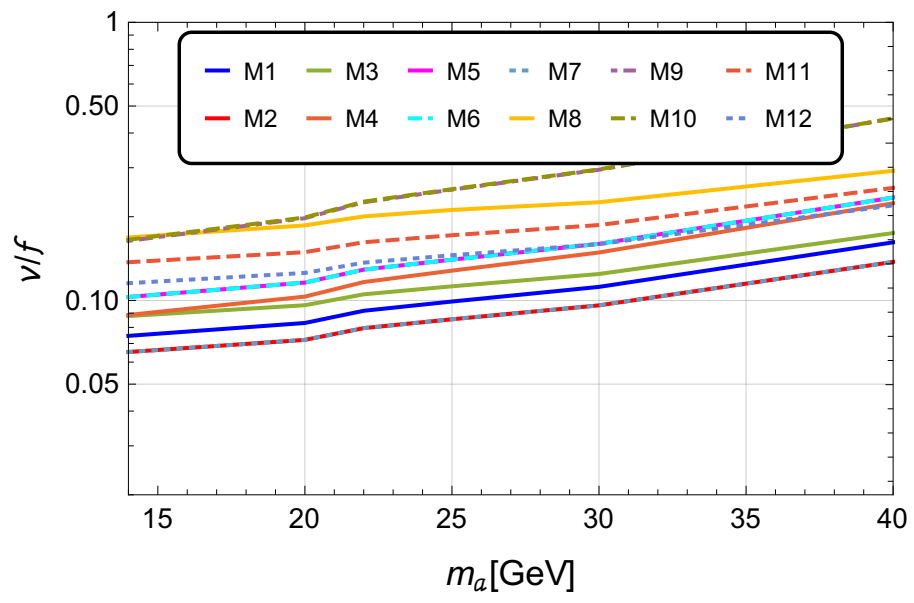

Figure 3. Projected sensitivity for integrated luminosity $L=15 \mathrm{fb}^{-1}$. Taken from [14].

ysis we see that muons still perform slightly better, but it should be remembered that the di-muon results come from a highly optimized experimental analysis. Also, di-tau resonances are interesting in their own right and would allow to probe models where $C_{\mu} \ll C_{\tau}$ accidentally.

\section{Conclusions}

In this short contribution I argued that realizing partial compositeness via ordinary 4D gauge theories provides a self contained concrete class of models to address the hierarchy problem. There are lots of open questions that go to the heart of strongly coupled theories, such as the range of the conformal window, anomalous dimensions and low energy coefficients, for which the lattice can provide invaluable information. These models are quite predictive, since many couplings can be computed from the underlying gauge theory. In particular, the presence of a ubiquitous light neutral pseudo-scalar $a$ can be tested in various decay channels such as di-muon or di-tau.

\section{Acknowledgments}

I wish to thank the organizers of this workshop for their kind invitation and their big effort in the face of the current difficult situation. I thank my colleagues Diogo Buarque Franzosi, 
Giacomo Cacciapaglia, Xabier Cid Vidal, Thomas Flacke and Carlos Vázquez Sierra for collaboration on many of the results presented in this talk. This work is supported by the Knut and Alice Wallenberg foundation under the grant KAW 2017.0100 (SHIFT project).

\section{References}

[1] D.B. Kaplan, H. Georgi, Phys. Lett. 136B, 183 (1984)

[2] N. Arkani-Hamed, A.G. Cohen, E. Katz, A.E. Nelson, JHEP 07, 034 (2002), hep-ph/0206021

[3] D.B. Kaplan, Nucl. Phys. B365, 259 (1991)

[4] J. Barnard, T. Gherghetta, T.S. Ray, JHEP 02, 002 (2014), 1311.6562

[5] G. Ferretti, D. Karateev, JHEP 03, 077 (2014), 1312 . 5330

[6] G. Ferretti, JHEP 06, 107 (2016), 1604.06467

[7] A. Belyaev, G. Cacciapaglia, H. Cai, G. Ferretti, T. Flacke, A. Parolini, H. Serodio, JHEP 01, 094 (2017), 1610.06591

[8] V. Ayyar, T. Degrand, D.C. Hackett, W.I. Jay, E.T. Neil, Y. Shamir, B. Svetitsky, Phys. Rev. D 97, 114505 (2018), 1801.05809

[9] V. Ayyar, T. DeGrand, D.C. Hackett, W.I. Jay, E.T. Neil, Y. Shamir, B. Svetitsky, Phys. Rev. D 99, 094502 (2019), 1812 . 02727

[10] J.W. Lee, E. Bennett, D.K. Hong, C.J.D. Lin, B. Lucini, M. Piai, D. Vadacchino, PoS LATTICE2018, 192 (2018), 1811.00276

[11] E. Bennett, D.K. Hong, J.W. Lee, C.J.D. Lin, B. Lucini, M. Piai, D. Vadacchino, JHEP 12, 053 (2019), 1909. 12662

[12] G. Cacciapaglia, G. Ferretti, T. Flacke, H. Serodio, Eur. Phys. J. C 78, 724 (2018), 1710.11142

[13] G. Cacciapaglia, G. Ferretti, T. Flacke, H. Serôdio, Front. in Phys. 7, 22 (2019), 1902.06890

[14] D. Buarque Franzosi, G. Cacciapaglia, X. Cid Vidal, G. Ferretti, T. Flacke, C. Vázquez Sierra (2021), 2106. 12615

[15] R. Aaij et al. (LHCb), JHEP 10, 156 (2020), 2007.03923

[16] U. Haisch, J.F. Kamenik, Phys. Rev. D 93, 055047 (2016), 1601.05110

[17] U. Haisch, J.F. Kamenik, A. Malinauskas, M. Spira, JHEP 03, 178 (2018), 1802 . 02156 